Crystallization in suspensions of hard spheres: a Monte Carlo and molecular dynamics simulation study

This article has been downloaded from IOPscience. Please scroll down to see the full text article.

2011 J. Phys.: Condens. Matter 23194120

(http://iopscience.iop.org/0953-8984/23/19/194120)

View the table of contents for this issue, or go to the journal homepage for more

Download details:

IP Address: 158.64.77.122

The article was downloaded on 29/07/2013 at 14:21

Please note that terms and conditions apply. 


\title{
Crystallization in suspensions of hard spheres: a Monte Carlo and molecular dynamics simulation study
}

\author{
T Schilling ${ }^{1}$, S Dorosz ${ }^{2}$, H J Schöpe ${ }^{2}$ and G Opletal ${ }^{3}$ \\ ${ }^{1}$ Theory of Soft Condensed Matter, Université du Luxembourg, Luxembourg, Luxembourg \\ ${ }^{2}$ Institut für Physik, Johannes Gutenberg-Universität Mainz, D-55099 Mainz, Germany \\ ${ }^{3}$ School of Applied Sciences, RMIT University, Melbourne, Australia
}

Received 30 September 2010, in final form 17 November 2010

Published 27 April 2011

Online at stacks.iop.org/JPhysCM/23/194120

\begin{abstract}
The crystallization of a metastable melt is one of the most important non-equilibrium phenomena in condensed matter physics, and hard sphere colloidal model systems have been used for several decades to investigate this process by experimental observation and computer simulation. Nevertheless, there is still an unexplained discrepancy between the simulation data and experimental nucleation rate densities. In this paper we examine the nucleation process in hard spheres using molecular dynamics and Monte Carlo simulation. We show that the crystallization process is mediated by precursors of low orientational bond-order and that our simulation data fairly match the experimental data sets.
\end{abstract}

(Some figures in this article are in colour only in the electronic version)

\section{Introduction}

Hard spheres are often used as a model system to study the liquid to crystal transition. More than fifty years ago the existence of the freezing transition in hard spheres had already been shown by computer simulation methods [1]. As the interaction potential between two hard spheres is infinite when they overlap and zero otherwise, the phase behaviour is determined only by entropy rather than by a competition between enthalpy and entropy. The simple interaction potential makes hard spheres a particularly popular model system for computer simulation studies of crystallization and the competing glass transition (see e.g. [2-10]).

Hard sphere-like systems have also been realized experimentally in colloidal suspensions since the 1980s [11]. Using scattering techniques as well as microscopy, the crystallization process and the competing glass transition have been studied in detail during the past decade (see e.g. [12-16]).

The recent interest in studying the crystallization process of hard spheres using computer simulation has been triggered in particular by the following reasons.

Crystal nucleation from a supersaturated liquid is a typical 'rare event'. It occurs (by definition) after an induction time that is much longer than the timescale for thermalization of the microscopic degrees of freedom of the system, and it changes the properties of the system drastically. Computer simulation of rare events requires special techniques in order to avoid wasting large amounts of CPU time on irrelevant microscopic fluctuations. For the past decade crystal nucleation has been commonly used as an example problem to apply rare event sampling techniques. However, in the meantime computers have become fast enough to sample crystal nucleation by 'brute force' simulation in simple model systems, such as hard spheres. Hence hard spheres have recently been used to test the predictions of rare event sampling techniques (such as results obtained by Umbrella Sampling [3]) and to compare nucleation pathways directly to experiment $[6,10]$.

New experiments as well as simulations show deviations from the classical picture of crystallization, indicating that crystallization in hard sphere systems starts with the formation of precursors (low symmetry clusters, medium range ordered crystals) before real crystals are formed $[6,8,16,17]$. Similar observations have been made studying crystal nucleation in atomic systems using dynamical density functional theory [18]. Furthermore it was suggested that these precursors are linked with structural and dynamical heterogeneities of the metastable melt and that the formation of precursors might be linked with the glass transition $[19,20]$. Hence the topic of crystallization in hard spheres is currently being revisited within computer simulation studies. 
We have recently published results on the crystallization mechanism in hard spheres that were obtained by a brute force MC simulation [6]. Here we will add results on nucleation rates and a comparison of two types of microscopic dynamics with experimental data.

\section{Simulation details}

In order to test if the details of the short time dynamics affect the nucleation behaviour we have performed two types of simulation: event driven molecular dynamics (Newtonian free flight and collisions) and Monte Carlo simulations using only small translational moves (mimicking Brownian dynamics on long timescales [21, 22]).

In both cases we monitored crystallization by means of the $q 6 q 6$-bond order parameter [23, 24], the definition of which we briefly recapitulate: for a particle $i$ with $n(i)$ neighbours, the local orientational structure is characterized by

$$
\bar{q}_{l m}(i):=\frac{1}{n(i)} \sum_{j=1}^{n(i)} Y_{l m}\left(\vec{r}_{i j}\right),
$$

where $Y_{l m}\left(\vec{r}_{i j}\right)$ are the spherical harmonics corresponding to the orientation of the vector $\vec{r}_{i j}$ between particle $i$ and its neighbour $j$ in a given coordinate frame. We are interested in local fcc-, hcp- or rcp-structures. Therefore we consider $l=6$. A vector $\vec{q}_{6}(i)$ is assigned to each particle, the elements $m=-6-6$ of which are defined as

$$
q_{6 m}(i):=\frac{\bar{q}_{6 m}(i)}{\left(\sum_{m=-6}^{6}\left|\bar{q}_{6 m}(i)\right|\right)^{1 / 2}} .
$$

We counted particles as neighbours if their distance $r_{i j}<1.4 \sigma$ (where $\sigma$ is the particle diameter). Two neighbouring particles $i$ and $j$ were regarded as 'bonded' within a crystalline region if $\vec{q}_{6}(i) \cdot \vec{q}_{6}(j)>0.7$. We define $n_{\mathrm{b}}(i)$ as the number of 'bonded' neighbours of the $i$ th particle. If a particle has $n_{\mathrm{b}}>10$ (i.e. an almost perfectly hexagonally ordered surrounding), we call it 'crystalline'. A cluster of particles with $n_{\mathrm{n}}>5$ is named a low symmetry cluster (LSC).

For the Monte Carlo simulations we use 1000 'sweeps' (1000 attempted MC moves per particle) as units of time, for the MD one time unit ('step') corresponds to 27 collisions per particle on average.

The system sizes we simulated were $N=8000$, 14400, 64000, and 216000 particles. We studied three densities $N \sigma^{3} / V=1.0238$ (packing fraction $\eta=0.5361$ ), $N \sigma^{3} / V=1.0269(\eta=0.5377)$ and $N \sigma^{3} / V=1.03(\eta=$ 0.5393). These densities correspond to chemical potential differences between the metastable liquid and the stable, almost completely crystalline state of $\Delta \mu \simeq-0.54 k_{\mathrm{B}} T$, $\Delta \mu \simeq-0.56 k_{\mathrm{B}} T$ and $\Delta \mu \simeq-0.58 k_{\mathrm{B}} T$ respectively. The interfacial tension is of the order of $0.5 k_{\mathrm{B}} T / \sigma^{2}[25,26]$. Table 1 summarizes the simulation runs we have performed.

Overcompressed liquid configurations were prepared by a fast pressure quench from the equilibrated liquid. During the quench we monitored the crystallinity to ensure that no crystal precursors were formed. (As prestructuring during the preparation procedure can have a significant impact on
Table 1. Simulation details. For the case $\mathrm{MC}^{a}$ the rate was computed from the number of clusters in the system as a function of time, while all other rates were obtained from first-nucleation-event-times.

\begin{tabular}{lrlrl}
\hline Type & \multicolumn{1}{c}{$N$} & $N \sigma^{3} / V$ & Runs & Rate $\left(\sigma^{5} / D_{1}\right)$ \\
\hline MC & 8000 & 1.0269 & 4 & $3.00 \times 10^{-6}$ \\
MC & 64000 & 1.0269 & 6 & $4.05 \times 10^{-6}$ \\
MC & 216000 & 1.0269 & 2 & $3.67 \times 10^{-6}$ \\
MC & 8000 & 1.03 & 8 & $2.10 \times 10^{-5}$ \\
MC & 14400 & 1.03 & 4 & $2.75 \times 10^{-5}$ \\
MC & 64000 & 1.03 & 4 & $1.82 \times 10^{-5}$ \\
MC $^{a}$ & 216000 & 1.03 & 5 & $1.07 \times 10^{-5}$ \\
MC $^{a}$ & 216000 & 1.03 & 5 & $1.73 \times 10^{-5}$ \\
MD & 64000 & 1.0238 & 5 & $7.2 \times 10^{-7}$ \\
MD & 8000 & 1.0269 & 29 & $1.93 \times 10^{-6}$ \\
MD & 8000 & 1.03 & 7 & $4.5 \times 10^{-6}$ \\
\hline
\end{tabular}

the nucleation behaviour, we cross-checked the quality of our starting configurations; the authors of [10] ran trajectories from our starting configurations using their simulation code. Within the error bars we found no differences in the crystallization process observed.)

\subsection{Monte Carlo simulation}

The Monte Carlo simulations were performed at fixed $N, V$ and $T$ by small translational moves only. We let all systems evolve until they crystallized and sampled observables every 5000 sweeps. Then we prepared movies of the crystalline clusters and played them backwards in time. The moment when the stable crystallite was reduced to a cluster of about ten particles was recorded as the 'nucleation time' $t_{\mathrm{n}}$. (Apart from the systems of $N=216000$ particles, no system showed more than one crystallization event. In the case of $N=216000$, we used the time when the first crystallite formed, as well as the relation 'number of crystallites versus time' to extract the nucleation rate.) We also recorded the times when the last particle with $n_{\mathrm{b}}>10$ vanished ('last' when playing the movies backwards) and the time when the cluster shrank below 40 particles. For the observables we used to extract the crystallization rates and to discuss the crystallization mechanism, we found no difference between these criteria (apart from a slight shift of the timescale, obviously). Where times are indicated in the following, each simulation run has been shifted by $-t_{\mathrm{n}}$ setting the time to zero at the nucleation event.

\subsection{Molecular dynamics}

Molecular dynamics simulations were performed at constant $N, V$, and $E$. The initial velocities were drawn from a Gaussian distribution and the initial mean kinetic energy per particle was set to $3 / 2 k_{\mathrm{B}} T$. The total energy is constant over the time of the simulation since all interactions are elastic collisions following Newton's equations of motion. In between collisions, particles advance ballistically since no force is present. We employed an event driven molecular dynamics algorithm [27-30]. The analysis was done in the same way as for the MC simulation. 

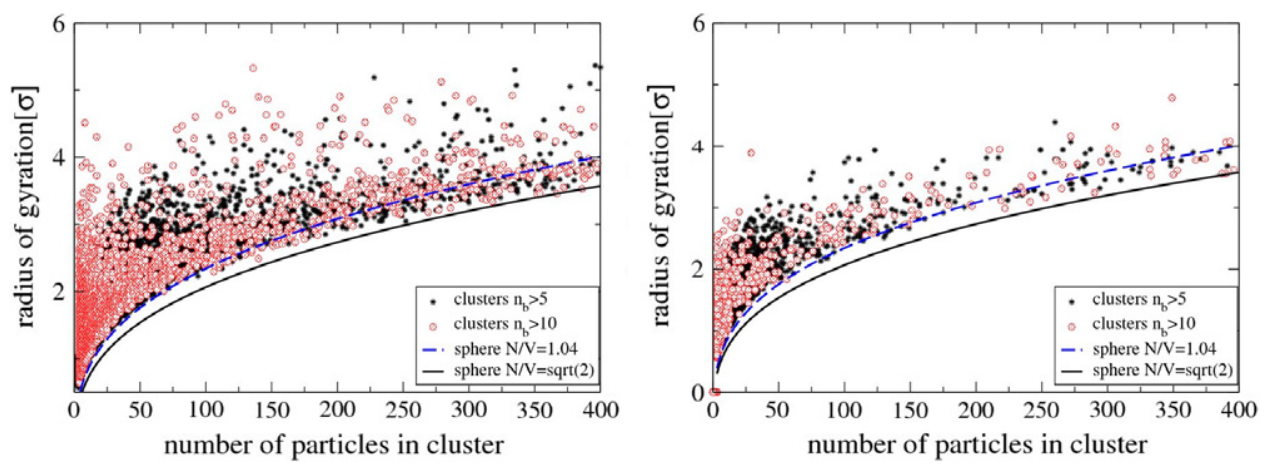

Figure 1. Radius of gyration versus number of particles in a cluster for $n_{\mathrm{b}}>5$ (stars) and $n_{\mathrm{b}}>10$ (circles, red online), $N \sigma^{3} / V=1.03$. Left panel: MC, right panel: MD. For comparison $R(N)$ is plotted for a sphere of $N \sigma^{3} / V=1.04$ (the density of a hard sphere crystal at coexistence, dashed line, blue online) and a sphere of $N \sigma^{3} / V=\sqrt{2}$ (solid line).
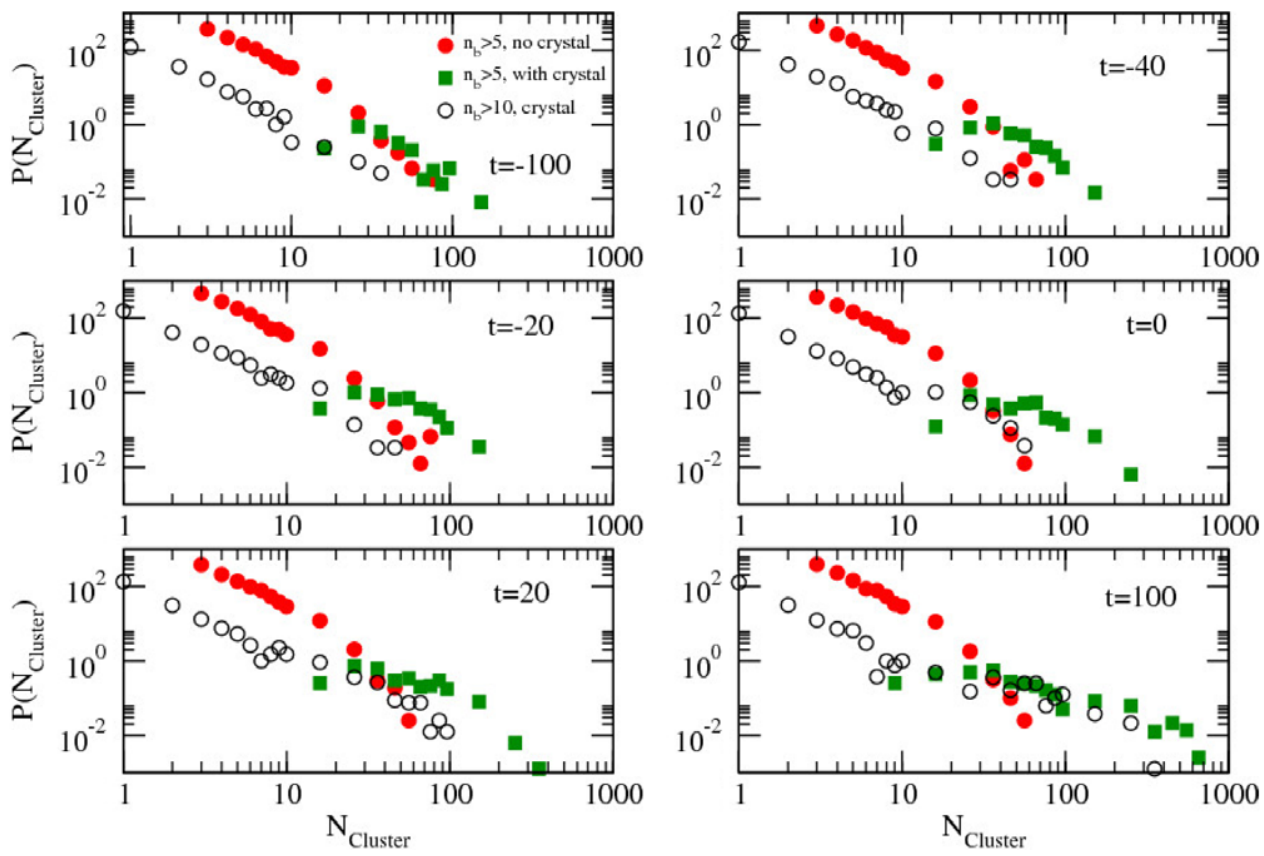

Figure 2. MC: distribution of cluster sizes for $n_{\mathrm{b}}>5$ containing no more than three particles with $n_{\mathrm{b}}>10$ (filled circles, red online), containing more than three particles with $n_{\mathrm{b}}>10$ (squares, green online) and for $n_{\mathrm{b}}>10$ (open circles). Times are given with respect to the time when the first stable crystallite appears (in units $1000 \mathrm{MC}$ sweeps). $N \sigma^{3} / V=1.03$.

\section{Results}

We first discuss the crystallite structures and then present results for the rates. Figure 1 shows the radius of gyration $R_{\mathrm{g}}$ versus the number of particles in a cluster $N_{\text {cluster }}$ for all clusters observed in the MC simulations (only up to 400 particles in a cluster to keep the graph readable). Stars indicate low symmetry clusters, circles crystalline clusters. In both the data from MC and from MD, there is a wide spread in $R_{\mathrm{g}}$; structures ranging from almost linear aggregates to very densely packed spheres occur. Even at large crystal sizes (i.e. in the crystal growth regime) there are ramified structures. Therefore, in the following discussion, we use the number of particles in a cluster rather than its radius to define a 'cluster size'.

Figure 2 shows the development of the cluster size distribution for the MC simulations. The data has been averaged over all simulation runs (shifted by the "nucleation time' as explained above). We distinguish between clusters of $n_{\mathrm{b}}>5$ with less than four particles that have $n_{\mathrm{b}}>$ 10 (i.e. 'empty' low symmetry clusters that do not contain crystallites, indicated by filled circles), clusters of $n_{\mathrm{b}}>5$ with 4 or more crystalline particles (squares) and clusters of particles with $n_{\mathrm{b}}>10$ (i.e. crystallites, open circles). The distribution of empty LSC does not vary with time. Just before crystallization sets in, at times $t=-40-0$, large low symmetry clusters that contain up to several hundred particles are formed, while the crystallites (empty circles) are still relatively small. Then the crystallites 'follow' until the two distributions coincide at $t=100$. This confirms our previous observation of a precursor mediated process [6].

Figure 3 shows the cluster size distributions for the MD simulation. As in the MC case, first low symmetry clusters 

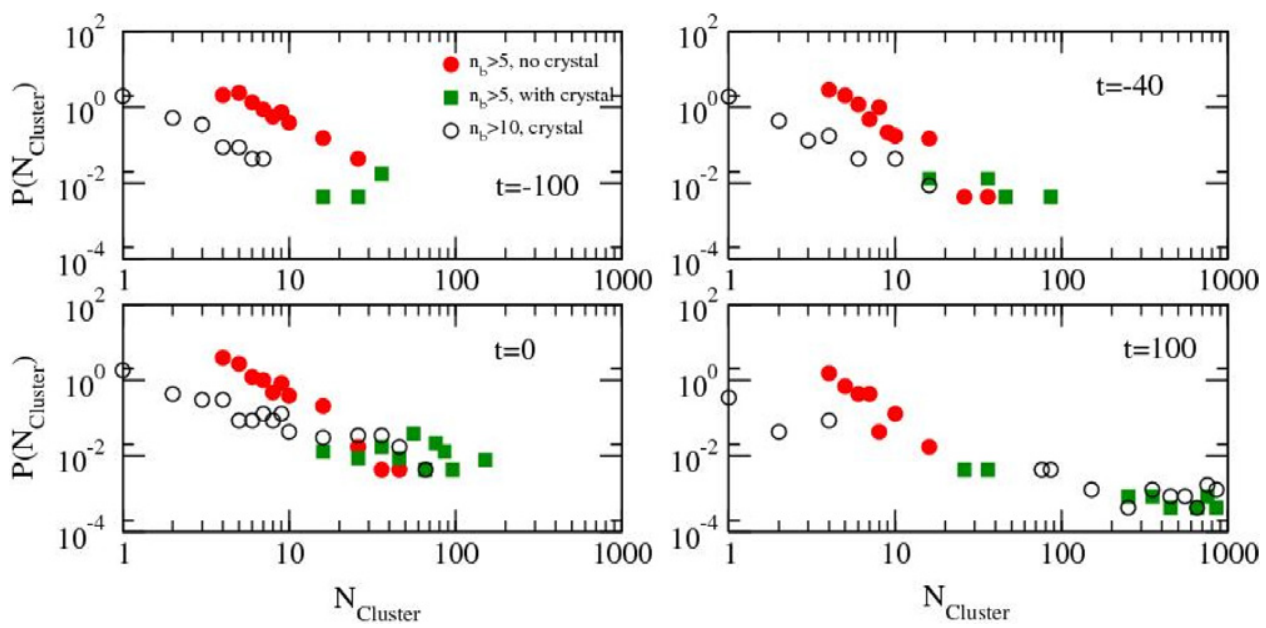

Figure 3. MD: distribution of cluster sizes for $n_{\mathrm{b}}>5$ containing no more than three particles with $n_{\mathrm{b}}>10$ (filled circles, red online), containing more than three particles with $n_{\mathrm{b}}>10$ (squares, green online) and for $n_{\mathrm{b}}>10$ (open circles). Times are given with respect to the time when the first stable crystallite appears (in units MD steps). $N \sigma^{3} / V=1.03$.

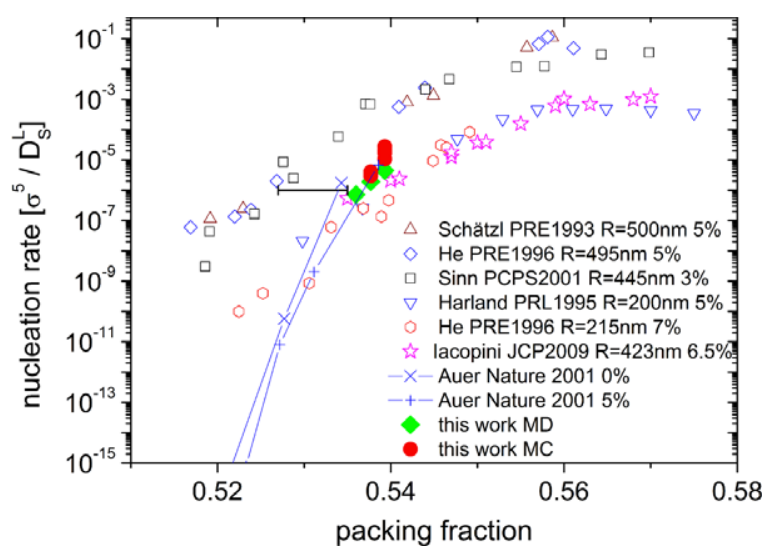

Figure 4. Nucleation rate density versus packing fraction. Solid symbols: data from this work; open symbols and crosses: experiments and simulations as cited and commented on in the main text.

form, then the crystallites appear. Hence the precursor-effect does not depend on the short time dynamics.

In figure 4 we compare the dimensionless nucleation rate density with experimental results and results from previous simulations. We scaled our data with the long time selfdiffusion coefficient $D_{1}$ extracted from our simulations. The typical error of our rate densities is about $50 \%$ To scale the experimental data we used the following expression of the long time self-diffusion coefficient provided by mode coupling theory $D_{\mathrm{l}} / D_{0}=\left(1-\eta / \eta_{\text {Glass }}\right)^{v}$ using $\eta_{\text {Glass }}=0.58$ and $v=2.6$ as determined in experiments. Please note that the data of [31-34] are scaled to the freezing volume fraction of monodisperse spheres, while the data of [17] are scaled to the freezing volume fraction of polydisperse spheres with $\sigma=6.5 \%$ polydispersity. The typical error in determining the volume fraction in these experiments is about \pm 0.004 as indicated by the horizontal error bar, while the error in the nucleation rate density is about one order of magnitude.
The experimental data fall onto two curves. This effect is probably due to differences in the methods by which the packing fraction was determined. However, considering the large uncertainty in the packing fraction these two curves still coincide within the error bars. The results obtained in our simulations (solid symbols, see also table 1) also agree with the experimental data within the error bars.

The simulation data of Auer and Frenkel [3] for samples with $5 \%$ polydispersity have been scaled to the freezing point of monodisperse spheres, allowing a direct comparison with the older experiments. Our data seem to lie below the simulation data from [3], but this effect might again still be within the error bars. It is not possible to extract an accurate statement regarding the slope of our data, as the interval of packing fractions we covered is relatively small. However, the slope is less steep than the slope of the Auer and Frenkel data for the monodisperse case and it is closer to the experiment.

We conclude that the previously observed discrepancy between simulation and experiment does not necessarily imply 'missing physics'. It can be explained by uncertainties and statistical errors.

In summary, we have presented a simulation study of crystallization in hard spheres. Both MD (Newtonian free flight and collisions) and MC (quasi-Brownian dynamics) show a precursor mediated crystallization process. First aggregates of low orientational bond-order form, then crystallites grow inside these. The shapes of the crystallites range from ramified structures to almost perfectly packed spheres. The crystallization rates agree with the experimental data as well as between MC and MD within the error bars.

\section{Acknowledgments}

We thank Laura Filion for cross-checking start configurations and Martin Oettel for fruitful discussions. This project has been financially supported by the DFG (SFB Tr6 and SPP1296). 


\section{References}

[1] Alder B J and Wainwright T E 1957 J. Chem. Phys. 271208

[2] Truskett T, Torquato S, Sastry S, Debenedetti P and Stillinger F 1998 Phys. Rev. E 583083

[3] Auer S and Frenkel D 2001 Nature 4091020

[4] Gruhn T and Monson P 2001 Phys. Rev. E 64061703

[5] O'Malley B and Snook I 2003 Phys. Rev. Lett. 90085702

[6] Schilling T, Schoepe H J, Oettel M, Opletal G and Snook I 2010 Phys. Rev. Lett. 105025701

[7] Zaccarelli E, Valeriani C, Sanz E, Poon W C K, Cates M E and Pusey P N 2009 Phys. Rev. Lett. 103135704

[8] Kawasaki T and Tanaka H 2010 Proc. Natl Acad. Sci. USA 10714036

[9] Charbonneau P, Ikeda A, van Meel J A and Miyazaki K 2010 Phys. Rev. E 81040501

[10] Filion L, Hermes M, Ni R and Dijkstra M 2010 Crystal nucleation of hard spheres using molecular dynamics, umbrella sampling and forward flux sampling: a comparison of simulation techniques arXiv:1006.2925v1 [cond-mat.soft]

[11] Pusey P and van Megen W 1986 Nature 320340

[12] Kegel W K and Dhont J K G 2000 J. Chem. Phys. 1123431

[13] Gasser U, Weeks E R, Schofield A, Pusey P N and Weitz D A 2001 Science 292258

[14] Weeks E and Weitz D 2002 Phys. Rev. Lett. 89095704

[15] Cheng Z, Chaikin P, Zhu J, Russel W B and Meyer W 2002 Phys. Rev. Lett. 88015501

[16] Schöpe H, Bryant G and van Megen W 2006 Phys. Rev. Lett. 96175701

[17] Iacopini S, Palberg T and Schöpe H 2009 J. Chem. Phys. 130084502
[18] Toth G, Tegze G, Pusztai T, Toth G and Granasy L 2010 J. Phys.: Condens. Matter 22364101

[19] Schöpe H, Bryant G and van Megen W 2007 J. Chem. Phys. 127084505

[20] Kawasaki T and Tanaka H 2010 J. Phys.: Condens. Matter 22084505

[21] Berthier L and Kob W 2007 J. Phys.: Condens. Matter 19205130

[22] Sanz E and Marenduzzo D 2010 J. Chem. Phys. 132194102

[23] Steinhardt P J, Nelson D R and Ronchetti M 1983 Phys. Rev. B 28784

[24] ten Wolde P R, Ruiz-Montero M J and Frenkel D 1995 Phys. Rev. Lett. 752714

[25] Davidchack R L and Laird B B 2000 Phys. Rev. Lett. 854751

[26] Davidchack R L, Morris J R and Laird B B 2006 J. Chem. Phys. 125094710

[27] Alder B J and Wainwright T E 1959 J. Chem. Phys. 31459

[28] Marín M and Cordero P 1995 Comput. Phys. Commun. 92214

[29] Krantz A T 1996 ACM Trans. Model. Comput. Simul. 6185

[30] Lubachevsky B D 1991 J. Comput. Phys. 94255

[31] Schätzel K and Ackerson B 1993 Phys. Rev. E 483766

[32] Harland J L, Henderson S I, Underwood S M and van Megen W 1995 Phys. Rev. Lett. 753572

[33] Sinn C, Heymann A, Stipp A and Palberg T 2001 Trends in Colloid and Interface Science XV; 14th Conf. of the Eur. Soc. of the Colloid and-Interface Soc.(Patras, Sept. 2000) vol 118 Progress in Colloid and Polymer Science ed P G Koutsoukos (Berlin: Springer) pp 266-75, ISBN 3-540-42241-2

[34] He Y, Ackerson B J, van Megen W, Underwood S M and Schtzel K 1996 Phys. Rev. E 545286 